\title{
ON TWO POINT DISTORTION THEOREMS FOR BOUNDED UNIVALENT REGULAR FUNCTIONS
}

\author{
JAMES A. Jenkins
}

1. Let $f(z)$ be a bounded univalent regular function mapping the unit disc $D$ into the unit disc $E$. We define

$$
\Delta_{1} f(z)=\frac{\left(1-|z|^{2}\right)}{\left(1-|f(z)|^{2}\right)} f^{\prime}(z) .
$$

The expression $\left|\Delta_{1} f(z)\right|$ is invariant under linear transformations of $D$ and of $E$. For $z_{1}, z_{2} \in D$ distinct let $\rho$ be the hyperbolic distance between $z_{1}$ and $z_{2}$ and $\sigma$ the hyperbolic distance between $f\left(z_{1}\right)$ and $f\left(z_{2}\right)$. These are of course invariant under linear transformations of $D$ and $E$. A two point distortion theorem for $f$ is an inequality between $\left|\Delta_{1} f\left(z_{1}\right)\right|,\left|\Delta_{1} f\left(z_{2}\right)\right|, \rho$ and $\sigma$. To prove such a result it is then sufficient to prove it for a suitable normalization for $z_{1}, z_{2}, f\left(z_{1}\right)$ and $f\left(z_{2}\right)$.

Many years ago Blatter [1] gave a similar result for univalent functions in $D$ (not satisfying a boundedness condition) namely

$$
\left|f\left(z_{1}\right)-f\left(z_{2}\right)\right|^{2} \geq \frac{\sinh 2 \rho}{8 \cosh 4 \rho} \sum_{j=1}^{2}\left(1-\left|z_{j}\right|^{2}\right)^{2}\left|f^{\prime}\left(z_{j}\right)\right|^{2} .
$$

Kim and Minda [5] pointed out that the first factor on the right is incorrect and extended the result to obtain

$$
\left|f\left(z_{1}\right)-f\left(z_{2}\right)\right| \geq \frac{\sinh 2 \rho}{2(2 \cosh 2 p \rho)^{1 / p}}\left(\left|D_{1} f\left(z_{1}\right)\right|^{p}+\left|D_{1} f\left(z_{2}\right)\right|^{p}\right)^{1 / p}
$$

where $D_{1} f(z)=\left(1-|z|^{2}\right) f^{\prime}(z)$ valid for $p \geq P$ with some $P, 1<P \leq 3 / 2$. In each case there was an appropriate equality statement.

Recently the author [4] has proved that [2] is valid for all $p \geq 1$ and also has given an inequality in the opposite direction.

Ma and Minda [6] have given for bounded univalent regular functions upper and lower bounds for $\sigma$ in terms of $\left|\Delta_{1} f\left(z_{1}\right)\right|,\left|\Delta_{1} f\left(z_{2}\right)\right|$ and $\rho$ depending on a

Received January 13, 2000; revised April 26, 2001. 
parameter $p$ conditioned by $p \geq 3 / 2$. Their proof is based on estimates for length in the hyperbolic metric and results in extremely complicated expressions in $\left|\Delta_{1} f\left(z_{1}\right)\right|,\left|\Delta_{1} f\left(z_{2}\right)\right|$ and $\rho$.

In this paper we will obtain two point distortion theorems for bounded univalent regular functions more analogous to those in [4] which are more truly distortion theorems.

2. The proof in [4] was carried out by studying the family $\mathscr{F}$ of functions $f$ regular and univalent in $D$ satisfying, for $0<r<1, f(-r)=-1, f(r)=1$. Of course $\mathscr{F}$ depends on $r$ but this is kept fixed. The treatment consists of proving two theorems stated here as Theorem $\mathrm{T}$ and Theorem $\mathrm{F}$.

THEOREM T. If $f \in \mathscr{F}$ and $p \geq 1$

$$
\left(\left|f^{\prime}(-r)\right|^{p}+\left|f^{\prime}(r)\right|^{p}\right)^{1 / p} \leq \frac{4(\cosh 2 p \rho)^{1 / p}}{\left(1-r^{2}\right) \sinh 2 \rho} .
$$

Equality occurs only for functions mapping $D$ on the plane slit along a ray on the positive or negative real axis.

THEOREM F. If $f \in \mathscr{F}$ and $p>0$

$$
\left(\left|f^{\prime}(-r)\right|^{p}+\left|f^{\prime}(r)\right|^{p}\right)^{1 / p} \geq \frac{2^{1 / p} \cosh (\rho / 2)}{\cosh \rho} .
$$

Equality occurs only for a function $l_{0}$ mapping $D$ onto the plane slit along the real axis symmetrically through the point at infinity.

In the proof of Theorem $\mathrm{T}$ there is constructed a one-parameter family of functions $f_{b} \in \mathscr{F}$ which map $D$ onto an admissible domain [2] with respect to the quadratic differential

$$
\kappa_{1} \frac{(w-b)}{(w+1)^{2}(w-1)^{2}} d w^{2}
$$

where $\kappa_{1}$ real has the same sign as $b$ or exceptionally for $b=\infty$

$$
\kappa_{2} \frac{d w^{2}}{(w+1)^{2}(w-1)^{2}}
$$

with $\kappa_{2}<0$. The functions are determined explicitly and the proof is carried out by direct calculation. Incidentally the formula on p. 156 l.4 in [4] should read

$$
\frac{d}{d b} \log \left|f_{b}^{\prime}(r)\right|=-\frac{1}{b-1} \frac{1}{\left(b^{2}-1\right)^{1 / 2}} \log \left(\frac{1+r^{2}}{2 r} \frac{(b+1)^{1 / 2}-(b-1)^{1 / 2}}{(b+1)^{1 / 2}+(b-1)^{1 / 2}}\right)
$$

but the correct formula is used in the remainder of the proof. 
To treat the case of bounded univalent regular functions we construct functions analogous to the $f_{b}$. We fix $r, 0<r<1$, and $a, 0<a<r$, and denote by $\mathscr{G}$ the family of functions regular and univalent in $D$ with values in $E$ such that $g(-r)=-a, g(r)=a$.

There are three types of special functions in $\mathscr{G}$. In the first instance for $\psi$, $0<\psi<\pi$ the quadratic differential

$$
Q^{*}(w, \psi) d w^{2}=-\frac{\left(w-e^{-i \psi}\right)^{2}\left(w-e^{i \psi}\right)^{2}}{(w-a)^{2}(w+a)^{2}\left(w-a^{-1}\right)^{2}\left(w+a^{-1}\right)^{2}} d w^{2}
$$

is positive in $E$ and has the following trajectory structure. The open arcs on $|w|=1$ joining $e^{i \psi}$ and $e^{-i \psi}$ are trajectories and there is a further trajectory $\gamma$ in $E$ joining $e^{i \psi}$ and $e^{-i \psi}$ which divides $E$ into two circle domains for $Q^{*}(w, \psi) d w^{2}$ containing respectively the double poles $-a, a$. If we make two appropriate symmetric incisions from $e^{-i \psi}, e^{i \psi}$ along $\gamma$ into $E$ we obtain a domain $E_{\psi}$ which can be mapped conformally onto $D$ so that $-a, a$ go to $-r, r$. This induces on $D$ a quadratic differential

$$
Q(z, \phi) d z^{2}=-K^{-1} \frac{\left(z-e^{-i \phi}\right)^{2}\left(z-e^{i \phi}\right)^{2}}{(z-r)^{2}(z+r)^{2}\left(z-r^{-1}\right)^{2}\left(z+r^{-1}\right)^{2}} d z^{2}
$$

with a positive constant $K$. We denote the corresponding mapping from $D$ to $E_{\psi}$ by $h_{0}$. The values of $\phi$ fill an open arc $0<\phi_{0}<\phi<\pi-\phi_{0}, 0<\phi_{0}<\pi$.

A second type of special function is obtained by mapping $E$ into the $\zeta$-plane by the function

$$
l(w)=\frac{\left(1-a^{2}\right)^{2}}{a\left(1+a^{2}\right)} \frac{w}{(1+w)^{2}}+\frac{2 a}{1+a^{2}}
$$

$l(E)$ is the plane slit on the positive real axis from $\left(1+6 a+4 a^{4}\right) /\left(4 a\left(1+a^{2}\right)\right)$ to $\infty$ and $l(-a)=-1, l(a)=1$. Thus for $g \in \mathscr{G}, l g \in \mathscr{F}$. For

$$
b_{+}=\frac{1+6 r^{2}+r^{4}}{4 r\left(1+r^{2}\right)} \leq b \leq \frac{1+6 a^{2}+a^{4}}{4 a\left(1+a^{2}\right)}=\hat{b}
$$

the function $l^{-1} f_{b}$ is in $\mathscr{G}$ and the quadratic differential

$$
\lambda \frac{(\zeta-b)}{(\zeta+1)^{2}(\zeta-1)^{2}} d \zeta^{2}
$$

for a suitable $\lambda>0$ induces on $E$ a quadratic differential

$$
-\frac{(w-c)\left(w-c^{-1}\right)(w-1)^{2}}{(w-a)^{2}(w+a)^{2}\left(w-a^{-1}\right)^{2}\left(w+a^{-1}\right)^{2}} d w^{2}
$$

where $c=l^{-1}(b)$.

The third type of special function can be obtained by a similar construction 
with the function

$$
\tilde{l}(w)=\frac{\left(1-a^{2}\right)^{2}}{a\left(1+a^{2}\right)} \frac{w}{(1-w)^{2}}-\frac{2 a}{1+a^{2}}
$$

to obtain a function $\tilde{l}^{-1} f_{b}$ and a quadratic differential

$$
-\frac{(w-\hat{c})\left(w-\hat{c}^{-1}\right)(w+1)^{2}}{(w-a)^{2}(w+a)^{2}\left(w-a^{-1}\right)^{2}\left(w+a^{-1}\right)^{2}} d w^{2}
$$

with $\hat{c}=\tilde{l}^{-1} b$.

Combining these we have quadratic differentials $\hat{Q}(w, t) d w^{2}$ where

$$
\begin{array}{cc}
\hat{Q}(w, t)=-\frac{\left(w^{2}-2 t w+1\right)(w-1)^{2}}{(w-a)^{2}(w+a)^{2}\left(w-a^{-1}\right)^{2}\left(w+a^{-1}\right)^{2}}, & t^{*} \geq t \geq 1, \\
\hat{Q}(w, t)=-\frac{(w-2 t w+1)^{2}}{(w-a)^{2}(w+a)^{2}\left(w-a^{-1}\right)^{2}\left(w+a^{-1}\right)^{2}}, & 1 \geq t \geq-1, \\
\hat{Q}(w, t)=-\frac{\left(w^{2}-2 t w+1\right)^{2}}{(w-a)^{2}(w+a)^{2}\left(w-a^{-1}\right)^{2}\left(w+a^{-1}\right)^{2}}, & -1 \geq t \geq-t^{*}
\end{array}
$$

with $t^{*}=(1 / 2)\left(l^{-1} b_{+}+\left(l^{-1} b_{+}\right)^{-1}\right)$. Note that the definitions agree at $t=1,-1$. We denote the corresponding functions in $\mathscr{G}$ by $g_{t}, t^{*} \geq t \geq-t^{*}$.

Lemma 1. For $g \in \mathscr{G},\left|g^{\prime}(-r)\right|$ is maximized uniquely for $g_{t^{*}}$, minimized uniquely for $g_{-t^{*}},\left|g^{\prime}(r)\right|$ is maximized uniquely for $g_{-t^{*}}$, minimized uniquely for $g_{t^{*}}$.

$g_{t^{*}}(D)$ is an admissible domain for the quadratic differential

$$
-\frac{-(w-1)^{2} d w^{2}}{(w+a)^{2}\left(w+a^{-1}\right)^{2}(w-a)\left(w-a^{-1}\right)},
$$

$g g_{t^{*}}^{-1}$ is an admissible function for it. Applying the General Coefficient Theorem $[2,3]$ with $-a$ as $P_{1}$, we have the coefficients

$$
\alpha^{(1)}=-\frac{a^{2}}{2(1-a)^{2}\left(1+a^{2}\right)}, \quad a^{(1)}=\left(g^{\prime}(-r)\right)^{-1} g_{t^{*}}^{\prime}(-r) .
$$

The fundamental inequality gives

$$
-\frac{a^{2}}{2(1-a)^{2}\left(1+a^{2}\right)} \log \left|\frac{g_{t^{*}}^{\prime}(-r)}{g^{\prime}(-r)}\right| \leq 0
$$

or

$$
\left|g^{\prime}(-r)\right| \leq\left|g_{t^{*}}^{\prime}(-r)\right| .
$$

The equality statement follows from that in the General Coefficient Theorem. 
$g_{-t^{*}}(D)$ is an admissible domain for the quadratic differential

$$
\frac{(w+1)^{2} d w^{2}}{(w+a)^{2}\left(w+a^{-1}\right)^{2}(w-a)\left(w-a^{-1}\right)},
$$

$g g_{-t^{*}}^{-1}$ is an admissible function for it. Applying the General Coefficient Theorem with $-a$ as $P_{1}$ we have the coefficients

$$
\alpha^{(1)}=\frac{a^{2}}{2(1+a)^{2}\left(1+a^{2}\right)}, \quad a^{(1)}=\left(g^{\prime}(-r)\right)^{-1} g_{-t^{*}}^{\prime}(-r) .
$$

The fundamental inequality gives

$$
\frac{a^{2}}{2(1+a)^{2}\left(1+a^{2}\right)} \log \left|\frac{g_{t^{*}}^{\prime}(-r)}{g^{\prime}(-r)}\right| \leq 0
$$

or

$$
g^{\prime}(-r) \geq\left|g_{-t^{*}}^{\prime}(-r)\right|
$$

The equality statement follows from that in the General Coefficient Theorem.

The remaining statements follow by symmetry.

Lemma 2. The quantity $\left|g^{\prime}(-r)\right|^{p}+\left|g^{\prime}(r)\right|^{p}, p>0$, is maximized for a function $g_{t}$, uniquely up to translation along trajectories.

It is readily seen that $\left|g_{t}^{\prime}(-r)\right|,\left|g_{t}^{\prime}(r)\right|$ vary continuously with $t$ on $\left[-t^{*}, t^{*}\right]$ either by domain convergence or by the explicit expressions given below. For any $g \in \mathscr{G}$ there exists a $t \in\left[-t^{*}, t^{*}\right]$ with $\left|g^{\prime}(-r)\right|=\left|g_{t^{*}}^{\prime}(-r)\right|$. We apply the General Coefficient Theorem with the quadratic differential $\hat{Q}(w, t) d w^{2}$ for this value of $t$, the admissible domain $g_{t}(D)$ and the admissible function $g g_{t}^{-1}$. Taking $-r$ as $P_{1}, r$ as $P_{2}$ for $t \in\left[1, t^{*}\right]$ the corresponding coefficients are

$$
\begin{gathered}
\alpha^{(1)}=-\frac{a^{2}\left(a^{2}+2 t a+1\right)}{4(1-a)^{2}\left(1+a^{2}\right)^{2}}, \quad a^{(1)}=\left(g^{\prime}\left(-r^{-1}\right)\right)^{-1} g_{t}^{\prime}(-r), \\
\alpha^{(2)}=-\frac{a^{2}\left(a^{2}-2 t a+1\right)}{4(1+a)^{2}\left(1+a^{2}\right)^{2}}, \quad a^{(2)}=\left(g^{\prime}(r)\right)^{-1} g_{t}^{\prime}(r) .
\end{gathered}
$$

The fundamental inequality gives

$$
-\frac{a^{2}\left(a^{2}+2 t a+1\right)}{4(1-a)^{2}\left(1+a^{2}\right)^{2}} \log \left|\frac{g_{t}^{\prime}(-r)}{g^{\prime}(-r)}\right|-\frac{a^{2}\left(a^{2}-2 t a+1\right)}{4(1+a)^{2}\left(1+a^{2}\right)^{2}} \log \left|\frac{g_{t}^{\prime}(r)}{g^{\prime}(r)}\right| \leq 0
$$

thus $\left|g^{\prime}(r)\right| \leq\left|g_{t}^{\prime}(r)\right|$. Equality can occur only if $g$ is obtained from $g_{t}$ by translation along trajectories.

The other cases for $t$ are treated similarly. 
Lemma 3. $\left|g_{t}^{\prime}(-r)\right|$ decreases strictly monotonically as $t$ goes from $t^{*}$ to $-t^{*}$. $\left|g_{t}^{\prime}(r)\right|$ increases strictly monotonically as $t$ goes from $t^{*}$ to $-t^{*}$.

This follows at once by applying the preceding argument for two values of $t$ and Lemma 1.

3. In order to find the maximum of $\left|g_{t}^{\prime}(-r)\right|^{p}+\left|g_{t}^{\prime}(r)\right|^{p}$ for reasons of symmetry it is sufficient to consider $t$ in the interval $\left[0, t^{*}\right]$.

Lemma 4. For $t$ in the range of values $[0,1], p>0,\left|g_{t}^{\prime}(-r)\right|^{p}+\left|g_{t}^{\prime}(r)\right|^{p}$ decreases strictly monotonically as $t$ goes from 1 to 0 .

From (3) and (4) we have for corresponding values $z, w, t, \psi, \phi$ and $K>0$

$$
\begin{aligned}
& \frac{\left(z-e^{i \phi}\right)^{2}\left(z-e^{-i \phi}\right)^{2}}{(z+r)^{2}(z-r)^{2}\left(z+r^{-1}\right)^{2}\left(z-r^{-1}\right)^{2}} \\
& =K \frac{\left(w-e^{i \psi}\right)^{2}\left(w-e^{-i \psi}\right)^{2}}{(w+a)^{2}(w-a)^{2}\left(w+a^{-1}\right)^{2}\left(w-a^{-1}\right)^{2}}\left(\frac{d w}{d z}\right)^{2} .
\end{aligned}
$$

Letting $z \rightarrow-r, w \rightarrow-a ; z \rightarrow r, w \rightarrow a$ respectively we have

$$
\begin{aligned}
& \frac{r^{2}\left(r^{2}+2 r \cos \phi+1\right)^{2}}{4\left(1-r^{2}\right)^{2}\left(1+r^{2}\right)^{2}}=K \frac{a^{2}\left(a^{2}+2 a \cos \psi+1\right)^{2}}{4\left(1-a^{2}\right)^{2}\left(1+a^{2}\right)^{2}} \\
& \frac{r^{2}\left(r^{2}-2 r \cos \phi+1\right)^{2}}{4\left(1-r^{2}\right)^{2}\left(1+r^{2}\right)^{2}}=K \frac{a^{2}\left(a^{2}-2 a \cos \psi+1\right)^{2}}{4\left(1-a^{2}\right)^{2}\left(1+a^{2}\right)^{2}}
\end{aligned}
$$

and dividing

$$
\frac{\left(r^{2}+2 r \cos \phi+1\right)}{\left(r^{2}-2 r \cos \phi+1\right)}=\frac{\left(a^{2}+2 a \cos \psi+1\right)}{\left(a^{2}-2 a \cos \psi+1\right)}
$$

Integrating explicitly $\int(-Q(z, \phi))^{1 / 2} d z$ and $\int\left(-Q^{*}(w, \psi)\right)^{2} d w$ with suitable normalizations we get

$$
\frac{1}{2} \frac{r\left(1+2 r \cos \phi+r^{2}\right)}{\left(1-r^{2}\right)\left(1+r^{2}\right)} \log \frac{z+r}{1+r z}-\frac{1}{2} \frac{r\left(1-2 r \cos \phi+r^{2}\right)}{\left(1-r^{2}\right)\left(1+r^{2}\right)} \log \frac{r-z}{1-r z},
$$

and

$$
\frac{1}{2} \frac{a\left(1+2 a \cos \psi+a^{2}\right)}{\left(1-a^{2}\right)\left(1+a^{2}\right)} \log \frac{w+a}{1+a w}-\frac{1}{2} \frac{a\left(1-2 a \cos \psi+a^{2}\right)}{\left(1-a^{2}\right)\left(1+a^{2}\right)} \log \frac{a-w}{1-a w} .
$$


Comparing expansions about $-r,-a$ we find

$$
\begin{aligned}
\log (w+a)= & \log (z+r)-\log (1+r z)+\log (1+a w) \\
& -\frac{\left(1-2 r \cos \phi+r^{2}\right)}{\left(1+2 r \cos \phi+r^{2}\right)} \log \frac{r-z}{1-r z}+\frac{\left(1-2 a \cos \psi+a^{2}\right)}{\left(1+2 a \cos \psi+a^{2}\right)} \log \frac{a-w}{1-a w}
\end{aligned}
$$

so

$$
\begin{aligned}
\log g_{t}^{\prime}(-r)= & \frac{\left(1-2 r \cos \phi+r^{2}\right)}{\left(1+2 r \cos \phi+r^{2}\right)}\left(-\log \frac{2 r}{1+r^{2}}+\log \frac{2 a}{1+a^{2}}\right) \\
& -\log \left(1-r^{2}\right)+\log \left(1-a^{2}\right) .
\end{aligned}
$$

Similarly

$$
\begin{aligned}
\log g_{t}^{\prime}(r)= & \frac{\left(1+2 r \cos \phi+r^{2}\right)}{\left(1-2 r \cos \phi+r^{2}\right)}\left(-\log \frac{2 r}{1+r}+\log \frac{2 a}{1+a^{2}}\right) \\
& -\log \left(1-r^{2}\right)+\log \left(1-a^{2}\right) .
\end{aligned}
$$

Thus

$$
\begin{aligned}
\left(g_{t}^{\prime}(-r)\right)^{p}+\left(g_{t}^{\prime}(r)\right)^{p}= & \left(\frac{1-a^{2}}{1-r^{2}}\right)^{p}\left(\frac{2 a}{1+a^{2}} \frac{1+r^{2}}{2 r}\right)^{p \frac{1-2 r \cos \phi+r^{2}}{1+2 r \cos \phi+r^{2}}} \\
& +\left(\frac{1-a^{2}}{1-r^{2}}\right)^{p}\left(\frac{2 a}{1+a^{2}} \frac{1+r^{2}}{2 r}\right)^{p \frac{1+2 r \cos \phi+r^{2}}{1-2 r \cos \phi+r^{2}}} .
\end{aligned}
$$

A straightforward calculation shows that this decreases strictly monotonically as $t$ goes from 1 to 0 .

Corollary 1. To find the maximum of $\left|g_{t}^{\prime}(-r)\right|^{p}+\left|g_{t}^{\prime}(r)\right|^{p}, \quad p>0$, it is enough to consider the values $t \in\left[1, t^{*}\right]$.

For $t \in\left[1, t^{*}\right]$ the function $g_{t}^{\prime}(z)$ is given by $g_{t}^{\prime}(z)=(d / d z)\left(l^{-1} f_{b}(z)\right)$ where $t$, $b$ are corresponding values. Thus

$$
\begin{gathered}
g_{t}^{\prime}(r)=\frac{a(1+a)\left(1+a^{2}\right)}{(1-a)^{3}} f_{b}^{\prime}(r) \\
g_{t}^{\prime}(-r)=\frac{a(1-a)\left(1+a^{2}\right)}{(1+a)^{3}} f_{b}^{\prime}(-r)
\end{gathered}
$$

and to find the maximum of $\left|g_{t}^{\prime}(-r)\right|^{p}+\left|g_{t}^{\prime}(r)\right|^{p}$ we find the maximum of

$$
\left(\frac{a(1-a)\left(1+a^{2}\right)}{(1+a)^{3}}\right)^{p}\left(f_{b}^{\prime}(-r)\right)^{p}+\left(\frac{a(1+a)\left(1+a^{2}\right)}{(1-a)^{3}}\right)\left(f_{b}^{\prime}(r)\right)^{p}
$$


on the appropriate interval for $b$. We consider its derivative

$$
\begin{aligned}
& p\left(\frac{a(1-a)\left(1+a^{2}\right)}{(1+a)^{3}}\right)^{p}\left(f_{b}^{\prime}(-r)\right)^{p-1} \frac{d f_{b}^{\prime}(-r)}{d b} \\
& +p\left(\frac{a(1+a)\left(1+a^{2}\right)}{(1-a)^{3}}\right)\left(f_{b}^{\prime}(r)\right)^{p-1} \frac{d f_{b}^{\prime}(r)}{d b} .
\end{aligned}
$$

The first term is negative the second positive.

Lemma 5. The ratio of the terms in (9) decreases as $b$ increases. Thus (8) has a unique maximum on $\left[b_{+}, \hat{b}\right]$. If

$$
p \geq \log \frac{1+r}{1-r}\left(\log \left(\frac{1-a}{1+a} \frac{1+r}{1-r}\right)\right)^{-1}
$$

the maximum occurs for $b_{+}$. Otherwise it occurs for $b \in\left(b_{+} \hat{b}\right]$. Thus if $(10)$ holds the maximum of $\left|g_{t}^{\prime}(-r)\right|^{p}+\left|g_{t}^{\prime}(r)\right|^{p}$ occurs for $t=t^{*}$.

A direct calculation shows that

$$
\begin{aligned}
& \frac{d}{d b} \frac{\left(f_{b}^{\prime}(-r)\right)^{p}(d / d b) f_{b}^{\prime}(-r)}{\left(f_{b}^{\prime}(r)\right)^{p-1}(d / d b) f_{b}^{\prime}(r)} \\
& \quad=\left(\frac{f_{b}^{\prime}(-r)}{f_{b}^{\prime}(r)}\right)\left(\frac{2}{(b+1)^{2}}\right)\left(\frac{p}{\left(b^{2}-1\right)^{1 / 2}}\right) \log \left(\frac{1+r^{2}}{2 r} \frac{(b+1)^{1 / 2}-(b-1)^{1 / 2}}{(b+1)^{1 / 2}+(b-1)^{1 / 2}}\right)-1
\end{aligned}
$$

which is negative (see [4], p. 156).

Therefore if $(8)$ is decreasing at $b_{+}$the maximal value occurs there. This requires the condition

$$
p>\log \frac{1+r}{1-r}\left(\log \left(\frac{1-a}{1+a} \frac{1+r}{1-r}\right)\right)^{-1}
$$

and the equality follows by a passage to the limit.

Using the invariance properties $\left|\Delta_{1} f(z)\right|, \rho$ and $\sigma$ and the expression for $\rho$ and $\sigma$ in terms of $r$ and $a$

$$
\rho=\log \frac{1+r}{1-r}, \quad \sigma=\log \frac{1+a}{1-a}
$$

we have the following theorem.

THEOREM 1. If $f$ is regular and univalent in $D$ and maps $D$ into $E$ and if $z_{1}, z_{2}$ are distinct points in $D$ and

$$
p \geq \frac{\rho}{\rho-\sigma}
$$


where $\rho, \sigma$ are the hyperbolic distances between $z_{1}$ and $z_{2}, f\left(z_{1}\right)$ and $f\left(z_{2}\right)$ respectively then

$$
\begin{aligned}
\left|\Delta_{1} f\left(z_{1}\right)\right|^{p}+\left|\Delta_{1} f\left(z_{2}\right)\right|^{p} \leq & {\left[\tanh \frac{\sigma}{2} \frac{e^{2 \sigma+1}}{\left(e^{\sigma+1}\right)^{2}}\left(\tanh \frac{\rho}{2} \frac{e^{2 \rho}+1}{\left(e^{\rho}+1\right)^{2}}\right)^{-1}\right]^{p} } \\
& \times\left[\left(\frac{e^{\rho}\left(e^{\sigma}+1\right)}{e^{\sigma}\left(e^{\rho}+1\right)}\right)^{4 p}+\left(\frac{e^{\sigma}+1}{e^{\rho}+1}\right)^{4 p}\right]
\end{aligned}
$$

Equality occurs if and only if $f$ maps $D$ onto $E$ slit along a ray on the hyperbolic line determined by $f\left(z_{1}\right)$ and $f\left(z_{2}\right)$.

If $p<\rho /(\rho-\sigma)$ this result does not obtain.

4. A bound in the opposite sense can be obtained immediately from Theorem $\mathrm{F}$. Let $m_{0}$ be the function in $\mathscr{G}$ mapping $D$ onto $E$ with equal rectilinear slits proceeding from $\pm 1 . \quad l_{0}$ is the function $\left(\left(1+r^{2}\right) / r\right)\left(z /\left(1+z^{2}\right)\right)$ and if $\tau(w)=\left(\left(1+a^{2}\right) / a\right)\left(w /\left(1+w^{2}\right)\right) \quad m_{0}$ is $\tau^{-1} l_{0}$. For any $g \in \mathscr{G}, \tau g \in \mathscr{F}$. Thus by Theorem $\mathrm{F}$ for $p>0$

$$
\left|(\tau g)^{\prime}(-r)\right|^{p}+\left|(\tau g)^{\prime}(r)\right|^{p} \geq\left(l_{0}^{\prime}(-r)\right)^{p}+\left(l_{0}^{\prime}(r)\right)^{p}=2\left(\frac{1-r^{2}}{r\left(1+r^{2}\right)}\right)^{p}
$$

and

$$
\left|g^{\prime}(-r)\right|^{p}+\left|g^{\prime}(r)\right|^{p} \geq 2\left(\frac{a\left(1+a^{2}\right)}{1-a^{2}} \cdot \frac{1-r^{2}}{r\left(1+r^{2}\right)}\right)^{p} .
$$

Moreover

$$
\left|\Delta_{1} g(-r)\right|^{p}+\left|\Delta_{1} g(r)\right|^{p} \geq 2\left(\frac{a\left(1+a^{2}\right)}{\left(1-a^{2}\right)^{2}} \frac{\left(1-r^{2}\right)^{2}}{r\left(1+r^{2}\right)}\right)^{p} .
$$

Equality occurs only for $m_{0}$.

Using the invariance properties we have proved the following theorem.

THEOREM 2. If $f$ is regular and univalent in $D$ mapping $D$ into $E$ and $z_{1}, z_{2}$ are distinct points of $D$, we have for $p>0$

$$
\left(\left|\Delta_{1} f\left(z_{1}\right)\right|^{p}+\left|\Delta_{1} f\left(z_{2}\right)\right|^{p}\right)^{1 / p} \geq \frac{e^{2 \sigma}+1}{e^{2 \rho}+1}\left(\frac{e^{\rho}+1}{e^{\sigma}+1}\right)^{3}\left(\frac{\cosh (\sigma / 2)}{\cosh (\rho / 2)}\right)^{4}
$$

where $\rho$ is the hyperbolic distance between $z_{1}$ and $z_{2}, \sigma$ is the hyperbolic distance between $f\left(z_{1}\right)$ and $f\left(z_{2}\right)$. Equality occurs only for the function mapping $D$ onto $E$ slit along symmetric rays on the hyperbolic line determined by $f\left(z_{1}\right)$ and $f\left(z_{2}\right)$. 


\section{REFERENCES}

[ 1 ] C. Blatter, Ein Verzerrungssatz für schlichte Funktionen, Comment. Math. Helv., 53 (1978), 651-659.

[2] J. A. Jenkins, Univalent Functions and Conformal Mapping, Ergeb. Math. Grenzgeb. 18, Springer-Verlag, Berlin, 1958.

[3] J. A. Jenkins, On normalization in the General Coefficient Theorem, Proc. Internat. Congr. Mathematicians (Stockholm, 1962), Inst. Mittag-Leffler, Djursholm, 1963, 347-350.

[ 4 ] J. A. Jenkins, On weighted distortion in conformal mapping II, Bull. London Math. Soc., 30 (1998), 151-158.

[5] S. Kim AND D. Minda, Two point distortion theorems for univalent functions, Pacific J. Math., 163 (1994), 137-157.

[6] W. Ma And D. Minda, Two point distortion theorems for bounded univalent functions, Ann. Acad. Sci. Fenn. Math., 22 (1997), 425-444.

DePaRTMENT OF MATHEMATICS

WASHINGTON UNIVERSITY

ST. LouIS, MO 63130

USA 